\title{
Photo-induced cavities in chalcogenide photonic crystals
}

\author{
Michael W. Lee ${ }^{1}$, Christian Grillet ${ }^{1}$, Snjezana Tomljenovic-Hanic ${ }^{1}$, Cameron L. C. Smith ${ }^{1}$, Christelle Monat ${ }^{1}$, \\ Darren Freeman $^{2}$, Steve Madden², Barry Luther-Davies ${ }^{2}$, Benjamin J. Eggleton ${ }^{1}$ \\ ${ }^{1}$ CUDOS, School of Physics, University of Sydney, Sydney, NSW 2006, Australia \\ Phone: +612 90365187, Fax: +612 93517726, Email: mlee@physics.usyd.edu.au \\ ( ${ }^{2}$ CUDOS, Laser Physics Centre, Australian National University, Canberra, ACT 0200, Australia)
}

\begin{abstract}
We demonstrate a photonic crystal (PC) cavity formed post-fabrication by locally modifying the refractive index of a chalcogenide PC by using the photosensitivity of the chalcogenide glass.
\end{abstract}

\section{Introduction}

2-dimensional planar photonic crystals (PCs), consisting of a thin high index dielectric slab patterned with a $2 \mathrm{D}$ periodic array of air holes, are a promising platform for compact optical devices. In particular, control of light in defects within PCs has a wide range of promising applications such as nonlinear all-optical switching, microfluidic sensing, slow light waveguideing and quantum information processing [1]. In this work we present a first demonstration of a novel post processing technique for creating defects within a PC by locally modifying the refractive index of a photosensitive chalcogenide glass. Previously, post tuning of existing PC defect structures such as waveguides [2] and cavities [3] has been demonstrated using chalcogenide glasses, however we present a demonstration of a defect which was introduced by photosensitive post processing. We demonstrate a photoinduced double heterostructure type cavity in a chalcogenide $\mathrm{PC}$ and present in situ measurements of the cavity formation using a tapered fibre evanescant coupling technique [2]. This technique affords some control over the defect properties by monitoring the defect in situ during its formation.

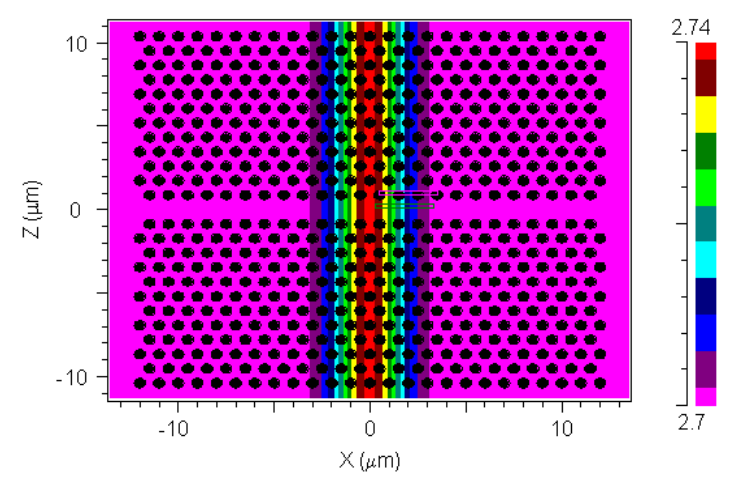

Figure 1. A schematic showing the index profile of a double heterostructure cavity formed by local index modification.

\section{Principle \& method}

Double heterostructure type cavities confine light by the 'mode gap' effect, and they are created by modifying a short section of a ' $\mathrm{W} 1$ ' waveguide in a way that locally increases the effective index of the waveguide. Typically this has been achieved by geometric modification of the PC lattice, e.g. increasing the lattice period of a short section of the waveguide [4], however, local modification of the refractive index by photosensitivity has also been proposed [5]. Figure 1 shows the required refractive index profile to create a double heterostructure type cavity by local index modification. The structure consists of a 'W1' waveguide with a short stripe of increased refractive index. Ideally this stripe should have a gaussian profile and the index contrast, $\Delta \mathrm{n}$, of $\sim 0.04$ to achieve the highest $Q$-factor.

The photonic crystal samples used for this work were 'W1' waveguides, i.e. waveguides formed by removal of a single row of holes from a hexagonal lattice. These samples were fabricated in a $300 \mathrm{~nm}$ thick membrane of AMTIR-1 $\left(\mathrm{Ge}_{33} \mathrm{As}_{12} \mathrm{Se}_{55}\right)$ chalcogenide glass and the array of holes was created by focused ion beam (FIB) milling [6]. The period of the structure was $520 \mathrm{~nm}$ and the hole radius was 0.3 time the period. The photosensitivity of the sample was characterized by the method presented in [2], and the refractive index was found to decrease when exposed to light at $633 \mathrm{~nm}$, the maximum refractive index change observed was $\sim 0.13$.

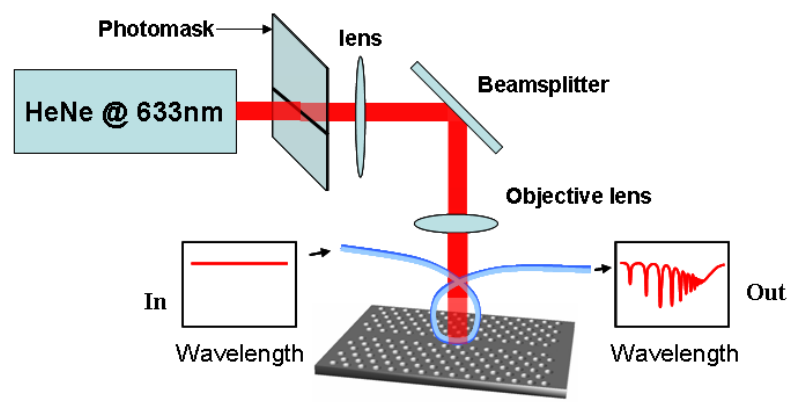

Figure 2. A schematic of our experimental setup. Selective exposure is achieved by using a photomask projected onto the sample and measurement is achieved by monitoring the transmission spectrum of a fibre taper in contact with the PC.

We performed our experiment using a setup which is represented schematically in figure 2 . This setup consists of two parts, the exposure system and the measurement system. The exposure system projects the image of a photomask onto sample to achieve selective exposure. A $633 \mathrm{~nm}$ HeNe laser with $6 \mathrm{~mW}$ power was used as the exposure light source. In this work a nominally $8 \mu \mathrm{m}$ 
wide dark stripe was projected onto the sample in order to achieve a similar index profile to figure 1 .

The measurement system consists of a tapered fibre, a broadband light source and an OSA. Measurements are performed by bringing the taper into contact with the PC sample and recording the taper's transmission spectrum. Dips in the transmission spectrum represent coupling to the modes of the PC. We used a looped taper with a $1.5 \mu \mathrm{m}$ waist diameter and an estimated contact length of $12 \mu \mathrm{m}$ with the waveguide. Spectra were recorded at 30 second intervals during the exposure.

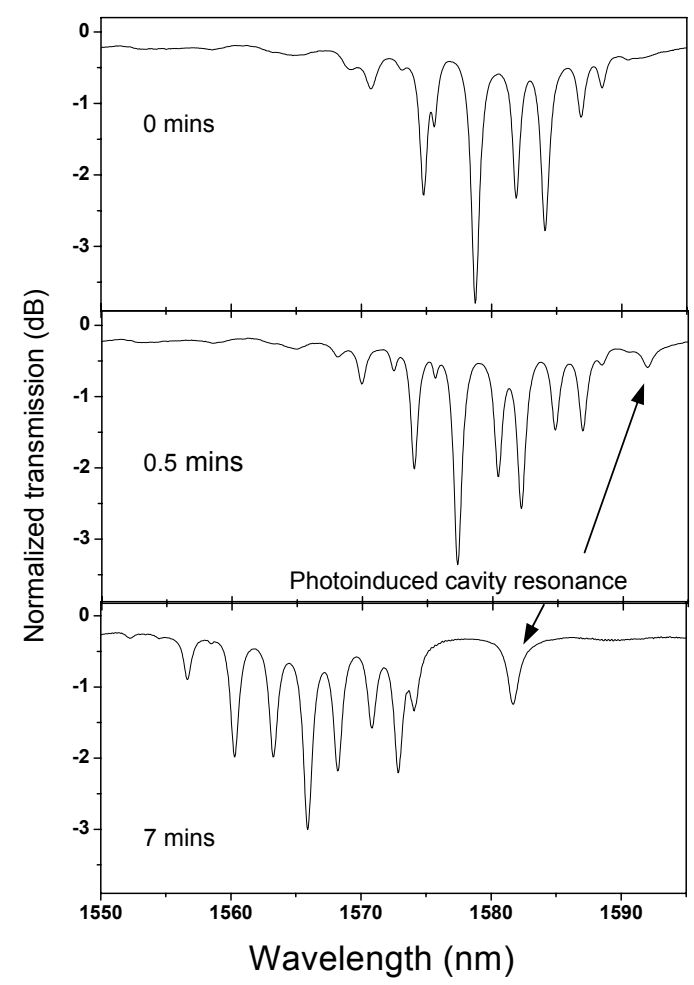

Figure 3. The measured coupling spectrum between the taper and $\mathrm{PC}$ waveguide at various times during the photosensitive experiment.

\section{Results \& discussion}

Figure 3 shows the spectra obtained at various times during the exposure. The initial spectrum (0 mins) shows a group of resonant features associated with the interaction between the taper and waveguide. We believe that these features are due to a taper induced cavity effect [7], where the presence of the taper itself causes the formation of localized modes. At 30 seconds into the exposure a new resonant feature was observed at longer wavelengths than the original group of features. We associate this feature with the photoinduced cavity mode. As the exposure progressed this feature became stronger and was seperated by up to $8 \mathrm{~nm}$ from the main group of features. The overall spectrum was observed to shift towards shorter wavelengths during the exposure due to the average decrease in refractive index due to exposure.
Further, we suspect that the dark stripe was not perfectly isolated from the exposing light by the photomask. Images of the sample under an optical microscope post exposure (figure 4) revealed a visible stripe of $\sim 3 \mu \mathrm{m}$ width instead of the $\sim 8 \mu \mathrm{m}$ width expected.

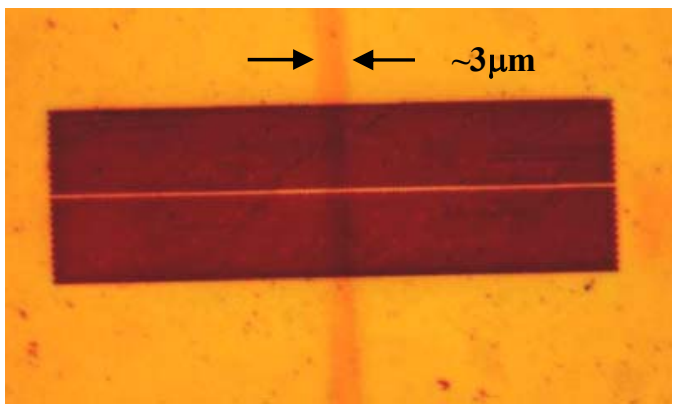

Figure 4. An optical microscope image of the PC waveguide post exposure. The shaded stripe is visible with a width of $\sim 3 \mu \mathrm{m}$.

The $Q$-factor obtained for the photoinduced resonance was $\sim 2000$. This value could have been limited by a number of factors, such as; a lower than expected index contrast between the exposed and shaded regions, parasitic degredation in $Q$-factor due to the presence of the taper in contact with the waveguide or material absorption issues related to the FIB milling of the sample and subsequent processing. In future we plan to investigate these issues further and expect to achieve higher $Q$-factors in subsequent experiments.

\section{Conclusions}

This work represents an encouraging first demonstration of a photoinduced defect in a chalcogenide planar photonic crystal. We were successfully able to induce a defect cavity into a PC waveguide using photosnesitivity. By employing an in situ monitoring technique we were able to observe the formation and growth of the cavity resonance during the exposure, affording a new degree of control over the final properties of the defect structure.

\section{References}

1. S. Noda, J. Lightwave Technol. 24, $4554-4567$ (2006)

2. M. W. Lee, C. Grillet, C. L. C. Smith, D. J. Moss, B. J. Eggleton, D. Freeman, B. Luther-Davies, S. Madden, A. Rode, Y. Ruan, and Y. -h. Lee, Opt. Express 15, 1277-1285 (2007)

3. A. Faraon, D Englund, D. Bulla, B. Luther-Davies, B. J. Eggleton, N, Stoltz, P. Petro, J. Vuckovic, Applied Physics Letters 92, 4 (2008)

4. B -S Song, S Noda, T Asano, Y Akahane, Nature Materials. 4, no. 3, 207-210 (2005)

5. S. Tomljenovic-Hanic, M. J. Steel, C. Martijn de Sterke, and D. J. Moss, Opt. Lett. 32, (2007)

6. D. Freeman, S. Madden, and B. Luther-Davies, Opt. Express 13, 3079-3086 (2005)

7. M. -K. Kim, I. -K. Hwang, M. -K. Seo, and Y. -H. Lee, Opt. Express 15, 17241-17247 (2007) 